\title{
A SIMPLE PROOF OF GABBER'S THEOREM ON PROJECTIVE MODULES OVER A LOCALIZED LOCAL RING
}

\author{
RICHARD G. SWAN
}

(Communicated by William C. Waterhouse)

\begin{abstract}
Let $A$ be a regular local ring of dimension 3 and let $u$ be an element of $A$ not in the square of the maximal ideal. Gabber has shown that all projective modules over $A\left[u^{-1}\right]$ are free. An elementary proof of this fact is given here.
\end{abstract}

In $[\mathbf{G}]$ Gabber proved the following result which confirms the first nontrivial case of a conjecture of Quillen [Q].

THEOREM (GABBER $[\mathbf{G}]$ ). Let $A$ be a regular local ring of dimension 3 with maximal ideal $\mathfrak{m}$. Let $u \in \mathfrak{m}-\mathfrak{m}^{2}$. Then all finitely generated projective $A_{u}$-modules are free.

Gabber's proof is rather complicated and makes use of nonabelian cohomology. I will give a very elementary proof here which follows the lines of Gabber's proof but avoids the technical difficulties.

I would like to thank M. P. Murthy for numerous discussions of Gabber's proof. Many of his ideas and suggestions have been incorporated into this paper. I would also like to thank C. Huneke for pointing out an error, discovered by T. Marley, in an earlier version of this paper.

All modules used in this paper will be assumed finitely generated.

1. Historical remarks. Quillen's conjecture arose in connection with the wellknown Bass-Quillen conjecture that all projective $R[T]$-modules are extended from $R$ if $R$ is regular. By [Q] it is enough to check this locally.

CONJECTURE $B Q_{n}$. If $R$ is a regular local ring of dimension $n$, every projective module over $R[T]$ is free.

This is known for $n \leq 2$ (Seshadri, Horrocks, Murthy, see [La]) and for geometric local rings $[\mathbf{L}]$. Quillen shows that it is enough to show that projective modules over $R(T)$ are free where $R(T)=R[T]_{S}, S=$ monic polynomials. Since $R(T)=A_{x}$ where $x=T^{-1}, A=R[x]_{(\mathfrak{m}, x)}$, this leads to Quillen's question.

CONJECTURE $Q_{n}$. If $A$ is a regular local ring of dimension $n$ and $u \in \mathfrak{m}-\mathfrak{m}^{2}$ then every projective $A_{u}$ module is free.

Since $\operatorname{dim} A=\operatorname{dim} R+1$, we see that $Q_{n+1}$ implies $B Q_{n}$. Therefore, unfortunately, Gabber's result does not imply any new cases of $B Q_{n} . Q_{n}$ is trivial for $n \leq 2$ since $A_{u}$ is a field for $n \leq 1$ and a principal ideal domain for $n=2$. The case where $A$ is geometric has been established by Bhatwadekar and Rao [BR].

Received by the editors October 31, 1986 and, in revised form, May 4, 1987.

1980 Mathematics Subject Classification (1985 Revision). Primary 13C10; Secondary 18G05, $13 \mathrm{H} 05$.

Supported in part by the National Science Foundation. 


\section{Reduction to the complete case.}

LEMMA 2.1. If $Q_{n}$ holds when $A$ is u-adically complete, it holds in general.

ProOF. Let $\hat{A}=\lim A / u^{m} A$ be the $u$-adic completion of $A$. This is also regular of dimension $n$ [Ma]. Clearly $A / u A \stackrel{\approx}{\rightarrow} \hat{A} / u \hat{A}$ and $u$ is regular on $A$ and $\hat{A}$. Under this condition, a number of authors have shown that the cartesian square

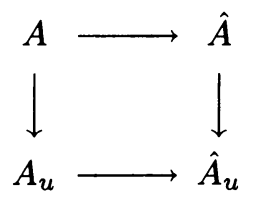

has the Milnor patching property. An expository treatment with references to some of the original papers may be found in [Sw]. Suppose $Q_{n}$ holds for $\hat{A}_{u}$. Let $P$ be projective over $A_{u}$. Then $\hat{A}_{u} \otimes_{A} P$ is free so we can patch $P$ with a free $\hat{A}$ module getting a projective $A$-module $Q$ such that $P=Q_{u}$. But $Q$ is free since $A$ is local.

COROLlARY. Let $R$ be a regular local ring. To check $B Q_{n}$ for $R$ it is enough to show that all projective modules over $R[[x]]\left[x^{-1}\right]$ are free.

3. Preliminary results. Let $M^{*}=\operatorname{Hom}_{R}(M, R) . M$ is reflexive if $M \stackrel{\approx}{\longrightarrow}$ $M^{* *}$. The following results are well known.

LEMMA 3.1 (CF. [Se]). If $R$ has global dimension $\leq 2$, all reflexive $R$-modules are projective.

We recall the proof for the reader's convenience and for further use. Choose a resolution

$$
R^{q} \rightarrow R^{p} \rightarrow M^{*} \rightarrow 0
$$

and take duals getting

$$
0 \rightarrow M \rightarrow R^{p} \rightarrow R^{q} \rightarrow X \rightarrow 0
$$

The lemma follows since $X$ has projective dimension $\leq 2$.

LEMMA 3.2. Let $R$ be a local ring and $M$ a reflexive $R$-module. Then, $\operatorname{depth} R \geq 2$ implies $\operatorname{depth} M \geq 2$.

PROOF. If $a \in \mathfrak{m}$ is regular on $R$ it is regular on $M=\operatorname{Hom}\left(M^{*}, R\right)$. Also, $\operatorname{Hom}\left(M^{*},-\right)$ on $0 \rightarrow R \rightarrow R \rightarrow R / a R \rightarrow 0$ gives $0 \rightarrow M / a M \rightarrow \operatorname{Hom}\left(M^{*}, R / a R\right)$ so if $b$ is regular on $R / a R$, it is regular on $M / a M$.

LEMMA 3.3. If $R$ is a domain, any $R$-module of the form $M=N^{*}$ is reflexive.

ProOF. The composition $N^{*} \stackrel{i}{\rightarrow} N^{* * *} \stackrel{j}{\rightarrow} N^{*}$ is the identity. Let $K$ be the quotient field of $R$. Since $K \otimes j$ is an isomorphism and $N^{* * *}$ is torsion free, $j$ is injective so $j$, and hence, $i$ is an isomorphism.

REMARK. It would be sufficient for $R$ to be reduced (take $K$ to be the total quotient ring) but some hypothesis on $R$ is needed. For example, the result is false for $R=A[x, y] /\left(x^{2}, x y, y^{2}\right)$ and $N=A$. 
LEMMA 3.4. Let $R$ be a domain, $M$ a reflexive $R$-module and $L \subset M$ a submodule. Then $L \subset L^{* *} \subset M$.

PROOF. The diagram

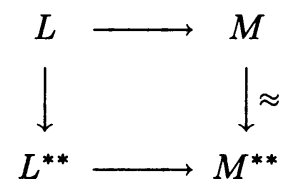

gives $L \rightarrow L^{* *} \rightarrow M$. Since all modules here are torsion free, injectivity of these maps follows by tensoring with the quotient field.

DEFINITION. If $\mathfrak{p}$ is a property of modules or diagrams over a local ring we say that $\mathfrak{p}$ nearly holds if $\mathfrak{p}$ holds after localization at any prime ideal other than the maximal ideal.

LEMMA 3.5. Let $f: X \rightarrow Y$ be nearly isomorphic. If $\operatorname{depth} R \geq 2$, then $f^{*}: Y^{*} \rightarrow X^{*}$ is an isomorphism.

ProOF. Write $0 \rightarrow K \rightarrow X \stackrel{j}{\rightarrow} I \rightarrow 0$ and $0 \rightarrow I \stackrel{i}{\rightarrow} Y \rightarrow Q \rightarrow 0$ where $f=j i$ and $I=\operatorname{im} f$. Then $K$ and $Q$ are of finite length, all composition factors being $R / \mathfrak{m}$ so $\operatorname{Hom}(K, R)=0, \operatorname{Ext}^{1}(K, R)=0$ and similarly for $Q$. The lemma now follows from the exact Ext sequences.

COROLLARY 3.6. If $Y$ in Lemma 3.5 is reflexive, then $Y \approx X^{* *}$ with $f$ corresponding to the canonical map $X \rightarrow X^{* *}$. In particular, if $X$ and $Y$ are reflexive, $f$ is an isomorphism.

This is clear from a diagram like (3).

LEMMA 3.7. Let $M$ have the form $M=N^{*}$. If $\operatorname{depth} R \geq 1$ and $M$ is nearly projective, then $M$ is reflexive.

ProOF. As in $3.3, N^{*}$ is a direct summand of $N^{* * *}$ so $M^{* *}=M \oplus X$. Since $M$ is nearly projective, $X$ has finite length and so is 0 since depth $M^{* *} \geq 1$ as in 3.2 .

LEMMA 3.8. If $\operatorname{depth} R \geq 2,0 \rightarrow M^{\prime} \rightarrow M \rightarrow M^{\prime \prime}$ is nearly exact, and $M^{\prime}, M, M^{\prime \prime}$ are nearly projective, then $0 \rightarrow M^{\prime * *} \rightarrow M^{* *} \rightarrow M^{\prime * *}$ is exact.

PROOF. Since $M \rightarrow M^{* *}$, etc., are near isomorphisms, the sequence in question is nearly exact, and the composition is 0 since the image of $M^{\prime * *}$ in $M^{\prime * * *}$ is of finite length. Let $X$ be the cokernel of $M^{\prime \prime *} \rightarrow M^{*}$. Then $0 \rightarrow X^{*} \rightarrow M^{* *} \rightarrow M^{\prime \prime * *}$ is exact so we get a map $M^{\prime * *} \rightarrow X^{*}$ which is a near isomorphism. Since $M^{\prime}$ is nearly projective, so are $X^{*}$ and $M^{\prime * *}$. Therefore these are reflexive by Lemma 3.7 and the result follows from Corollary 3.6.

DEFinition. Let $A$ be a local ring, $M$ a nearly projective $A$-module, and let $s \in$ $A$. Set $\Delta(M / s M)=(M / s M)^{* *}$ where the double dual is taken over the ring $A / s A$. The change of notation is intended to emphasize that results about $\Delta(M / s M)$ only apply to nearly projective modules and to make it clear where the duals are taken. We also define $H(M / s M)$ to be the cokernel of $M / s M \stackrel{i}{\rightarrow} \Delta(M / s M)$.

Since $M / s M$ is nearly projective, $i$ is a near isomorphism so $H(M / s M)$ is of finite length. The same is true of the kernel so $i$ is injective if $\operatorname{depth} M / s M \geq 1$. 
LEMMA 3.9. Let $A$ be a u-adically complete local ring where $u \in \mathfrak{m}$ is regular. Assume $\operatorname{depth} A \geq 3$. If $M$ is a reflexive $A$-module which is nearly projective then $M=\lim _{\longleftarrow} \Delta\left(M / u^{m} M\right)$.

ProOF. The sequence (1) above (with $A$ for $R$ ) is nearly split since $M^{*}$ is nearly projective. Therefore (2) is also nearly split so $0 \rightarrow M / u^{m} M \rightarrow\left(A / u^{m} A\right)^{p} \rightarrow$ $\left(A / u^{m} A\right)^{q}$ is nearly exact. By Lemma $3.8,0 \rightarrow \Delta\left(M / u^{m} M\right) \rightarrow\left(A / u^{m} A\right)^{p} \rightarrow$ $\left(A / u^{m} A\right)^{q}$ is exact. Taking limits gives $0 \rightarrow \lim \Delta\left(M / u^{m} M\right) \rightarrow A^{p} \rightarrow A^{q}$ and the result follows, the map of $M$ to the limit being given by $M \rightarrow M / u^{m} M \rightarrow$ $\Delta\left(M / u^{m} M\right)$.

4. Proof of Gabber's theorem. We assume $A$ to be $u$-adically complete by Lemma 2.1. Let $P$ be a projective $A_{u}$-module. We can assume rank $P \geq 2$ since $\operatorname{Pic}\left(A_{u}\right)=0$ (because $\mathbf{Z}=K_{0}(A) \rightarrow K_{0}\left(A_{u}\right)$ is onto by regularity). We will show that $P$ is decomposable and the theorem will follow by induction on rank $P$. Find a finitely generated $A$-module $M$ with $M_{u}=P$. By replacing $M$ by $M^{* *}$ we may assume, by Lemma 3.3, that $M$ is reflexive. If $\operatorname{dim} A \leq 2$ then $M$ is free by Lemma 3.1 proving $Q_{n}$ for $n \leq 2$. In our case, $\operatorname{dim} A=3$ so $M$ is nearly projective.

Following Gabber, we choose a reflexive $M$ with $M_{u}=P$ which is minimal in the sense that the length $h(M / u M):=l(H(M / u M))$ is as small as possible.

Since $R=A / u A$ is regular of dimension 2, Lemmas 3.3 and 3.1 show that $\Delta(M / u M)$ is free of $\operatorname{rank}=\operatorname{rank} P \geq 2$, so there is a nontrivial splitting $\Delta(M / u M)$ $=X \oplus Y$. Our aim is to lift this to a splitting of $M$ and therefore of $P$.

By Lemma 3.2, $\operatorname{depth} M \geq 2$ and so $\operatorname{depth} M / u M \geq 1$. Therefore $i: M / u M \rightarrow$ $\Delta(M / u M)=X \oplus Y$ is injective. Let $L$ and $N$ be the inverse images in $M$ of $X$ and $Y$. Then $L \cap N=u M$, and $L+N$ is nearly equal to $M$ since $i$ is nearly isomorphic, $M / u M$ being nearly projective over $R$. In other words,

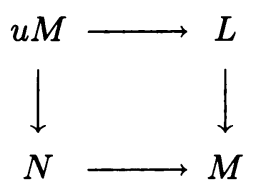

is nearly bicartesian.

LEMMA 4.1. L and $N$ are reflexive.

PrOOF. Locally at any prime $\mathfrak{p} \neq \mathfrak{m},(X \oplus Y)_{\mathfrak{p}}=M_{\mathfrak{p}} / u M_{\mathfrak{p}}$ has projective dimension 1 and therefore so do $X_{\mathfrak{p}}$ and $Y_{\mathfrak{p}}$. It follows from $0 \rightarrow L_{\mathfrak{p}} \rightarrow M_{\mathfrak{p}} \rightarrow Y_{\mathfrak{p}} \rightarrow 0$ that $L_{\mathfrak{p}}$ is projective so $L$ is nearly projective. Therefore $L^{* *} / L$ is of finite length. By Lemma 3.4, $L^{* *} / L \subset M / L \subset Y$. Since $Y$ is free over $R, \operatorname{depth} Y \geq 1$ so $L^{* *} / L=0$.

Consider the diagram

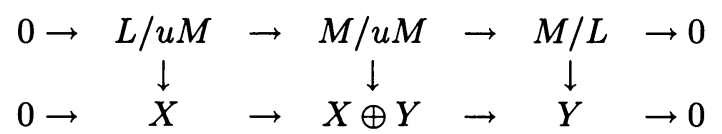

The vertical maps are injective and the lower sequence is $0 \rightarrow \Delta(L / u M) \rightarrow$ $\Delta(M / u M) \rightarrow \Delta(M / L) \rightarrow 0$ by Corollary 3.6. It follows that $0 \rightarrow H(L / u M) \rightarrow$ $H(M / u M) \rightarrow H(M / L) \rightarrow 0$ is exact. Writing $h(Z)=l(H(Z))$ as above, we have 
$h(M / u M)=h(L / u M)+h(M / L)$. Now consider the diagram

$$
\begin{array}{ccccccc}
0 \rightarrow & u M / u L & \rightarrow & L / u L & \rightarrow & L / u M & \rightarrow 0 \\
& \downarrow & & \downarrow & & \downarrow \\
0 \rightarrow & \Delta(u M / u L) & \rightarrow & \Delta(L / u L) & \stackrel{j}{\rightarrow} & \Delta(L / u M) &
\end{array}
$$

The bottom sequence is exact by Lemma 3.8 and the vertical maps are injective, the first and third being the same as in (4). This gives us

$$
0 \rightarrow H(u M / u L) \rightarrow H(L / u L) \rightarrow H(L / u M) \rightarrow Q \rightarrow 0
$$

where $Q=\operatorname{ckr} j$. Therefore $h(L / u L)+l(Q)=h(M / L)+h(L / u M)=h(M / u M)$. Since $M$ was minimal, it follows that $Q=0$ and $L$ is also minimal.

Since $j$ is onto, the bottom sequence splits because $\Delta(L / u M)=X$ is projective. We can now repeat the above construction with $L$ in place of $M$ and $\Delta(u M / u L)$ in place of $X$ getting a nearly bicartesian square

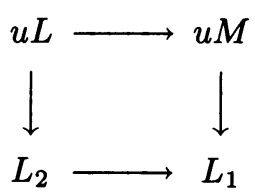

Write $L_{1}=L, N_{1}=N$ and iterate this construction to produce a diagram of nearly bicartesian squares

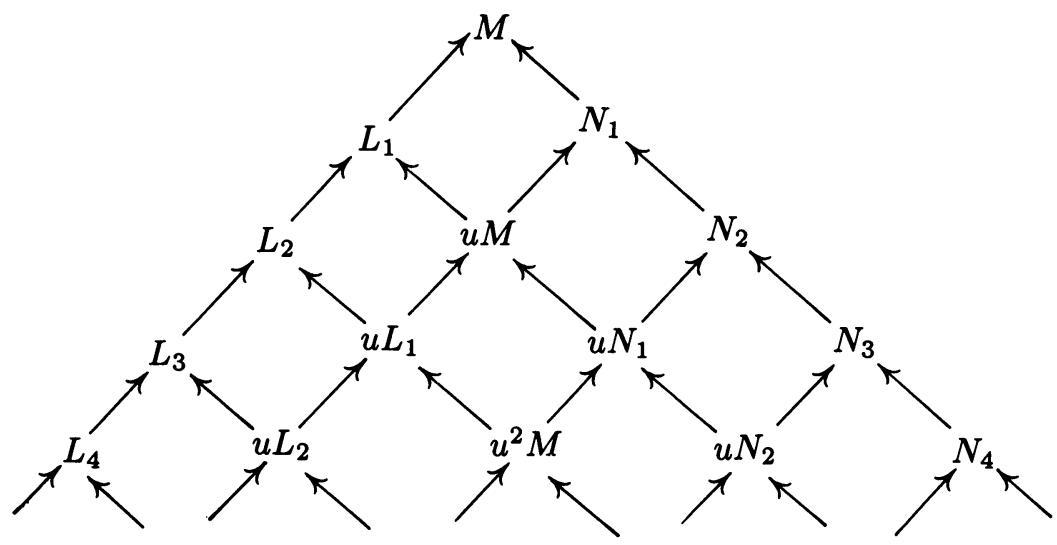

It follows that

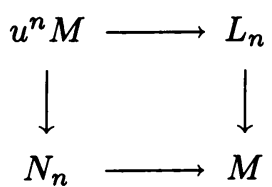

is nearly bicartesian so $L_{n} / u^{n} M \oplus N_{n} / u^{n} M \rightarrow M / u^{n} M$ is nearly isomorphic. Since $\operatorname{depth} A / u^{n} A \geq 2$, Lemma 3.5 gives

$$
\Delta\left(M / u^{n} M\right) \approx \Delta\left(L_{n} / u^{n} M\right) \oplus \Delta\left(N_{n} / u^{n} M\right)
$$

with all $\Delta$ 's taken over $A / u^{n} A$. Taking limits and using Lemma 3.9, we get the required splitting of $M$. It is nontrivial since the map of $M$ to $\Delta(M / u M)=X \oplus Y$ 
sends the summands into $\Delta(L / u M)=X$ and $\Delta(N / u M)=Y$. This completes the proof of the theorem.

REMARK (SEE [SGA2]). Let $X=\operatorname{Spec} R-\{\mathfrak{m}\}$. If $M$ is an $R$-module then $0 \rightarrow H_{\mathfrak{m}}^{0}(M) \rightarrow M \rightarrow \Gamma(X, \tilde{M}) \rightarrow H_{\mathfrak{m}}^{1}(M) \rightarrow 0$ and it follows that $M \stackrel{\approx}{\longrightarrow} \Gamma(X, \tilde{M})$ if $\operatorname{depth} M \geq 2$. If $\operatorname{depth} R \geq 2$ and $M$ is nearly projective then $\Gamma(X, \tilde{M})=M^{* *}$ since we can replace $M$ by $M^{* *}$ and use Lemma 3.2. Therefore $\Delta(M)=\Gamma(X, \tilde{M})$ as in Gabber's proof and $H(M)=H_{\mathfrak{m}}^{1}(M)$.

\section{BIBLIOGRAPHY}

[BR] S. M. Bhatwadekar and R. A. Rao, On a question of Quillen, Trans. Amer. Math. Soc. 279 (1983), 801-810.

[G] O. Gabber, Some theorems on Azumaya algebras, Groupes de Brauer, Lecture Notes in Math., vol. 844, Springer-Verlag, Berlin and New York, 1981, pp. 129-209.

[La] T. Y. Lam, Serre's conjecture, Lecture Notes in Math., vol. 635, Springer-Verlag, Berlin and New York, 1978.

[L] H. Lindel, On a question of Bass, Quillen, and Suslin concerning projective modules over polynomial rings, Invent. Math. 36 (1981), 319-323.

[Ma] H. Matsumura, Commutative algebra, Benjamin, New York, 1970.

[Q] D. Quillen, Projective modules over polynomial rings, Invent. Math. 36 (1976), 167-171.

[SG A2] A. Grothendieck, Cohomologie locale des faisceaux cohérents et théorèmes de Lefschetz locaux et globaux, North-Holland, Amsterdam, 1968.

[Se] J.-P. Serre, Classes des corps cyclotomiques, Sem. Bourbaki 11 (1958/59), Exp. 174.

[Sw] R. G. Swan, Projective modules over binary polyhedral groups, J. Reine Angew. Math. 342 (1983), 66-172.

Department of Mathematics, University of Chicago, Chicago, Illinois 60637 